Ein anderes Gesicht der Medizin - warum nicht ein Einsatz in Laos?

Bandi-Ott, E ; Busch, K ; Graf, A ; Stutz, B ; Zoller, M

Other titles: Muang Kham, Distriktspital - das Swiss Laos Hospital Projekt - das Team und die Einsätze

Posted at the Zurich Open Repository and Archive, University of Zurich

ZORA URL: https://doi.org/10.5167/uzh-81612

Journal Article

Published Version

Originally published at:

Bandi-Ott, E; Busch, K; Graf, A; Stutz, B; Zoller, M (2013). Ein anderes Gesicht der Medizin - warum nicht ein Einsatz in Laos? PrimaryCare, 13(18):331-333. 


\title{
Ein anderes Gesicht der Medizin - warum nicht ein Einsatz in Laos?
}

\author{
Muang Kham, Distriktspital - das Swiss Laos Hospital Projekt - das Team und die Einsätze
}

\section{Die Umstände}

Zur Behandlung der Diabetiker stehen Metformin und Daonil zur Verfügung - sonst nichts. Der kleine Distriktspital in Muang Kham verfügt seit vier Jahren über einen Anschluss ans Stromnetz. Infektionen des Gastrointestinaltraktes, der Lungen und der Harnwege sind häufig, Rickettsiosen und Meningitiden nicht selten und Tuberkulose eher selten, aber wichtig.

Das Arsenal der lokalen Schulmedizin ist auch häufigen Problemen wie dem «low back pain» gegenüber recht hilflos. Fälle schwerer Unterernährung sind zwar ein medizinisches Problem, aber nur über soziale Massnahmen nachhaltig behandelbar.

Unfallchirurgische Entscheide müssen ohne Radiologie getroffen werden. Bei den perinatalen Infektionen und bei gynäkologischen Eingriffen konnten grosse Fortschritte erzielt werden.

Die Qualitätskontrolle der vor 5 Jahren etablierten Laborgeräte ist nach wie vor unbefriedigend. Fortschritte in der Spitalhygiene werden auch durch Diebstahl und Korruption erschwert.

Soweit einige Stichworte zum Arbeitsumfeld in Muang Kham. Das Distriktspital liegt ca. eine Fahrstunde von der Provinzhauptstadt Phonsavan (ca. 60000 Einwohner) entfernt in einer nordöstlichen Provinz nahe der Grenze zu Vietnam im Hügelland. In der Ortschaft Muang Kham finden sich ein Markt, einige kleine Geschäfte, mehrere Restaurants, worunter zwei mit dem beliebten Karaokebetrieb und eine Kreuzung zweier asphaltierter Strassen. Die gesamte Szenerie ist recht ländlich, die Menschen sehen selten Ausländer und sind interessiert und freundlich.

Das gebirgige Binnenland Laos ist ein Vielvölkerstaat der Mekongregion. Es wurde im Vietnamkrieg massiv bombardiert, hat wenig Exportwirtschaft und Tourismus und zählt heute noch zu den ärmsten Ländern Südostasiens.

\section{Einige Fallbeispiele}

Eine 21-jährige Frau wird auf einem Traktoranhänger gebracht. Sie hat starke Bauchschmerzen, ist blass und schwach. Klinisch und mittels Ultraschall wird eine Eileiterschwangerschaft vermutet. Die Vorbereitungen ziehen sich etwas hin, aber die Patientin wird schliesslich operiert, hat postoperativ jedoch ein Hämoglobin von weniger als $5 \mathrm{~g} / \mathrm{dl}$. Blutkonserven müssen im Provinzspital bestellt werden, stehen erst am nächsten Tag zur Verfügung. Die Patientin überlebt.

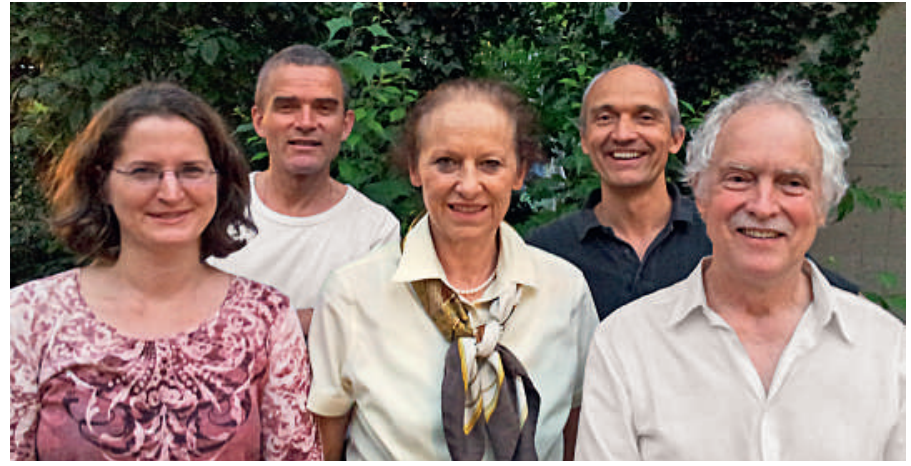

Abbildung 1

Kernteam v.l.n.r: Kerstin Busch, Beni Stutz, Elisabeth Bandi-Ott, Marco Zoller, Andreas Graf.

Eine 54-jährige Frau kann kaum mehr stehen, hat schmerzhafte Arthritiden mehrerer kleiner Gelenke, dünne Extremitäten und einen aufgetriebenen Bauch. Die ausführliche Befragung ergibt, dass sie seit Jahren wegen der Gelenkschmerzen ein billiges vietnamesisches Präparat einnimmt. Die Familie kann es schliesslich herbringen, es handelt sich um Dexamethason. Die Spitalapotheke verfügt über keinerlei Basismedikation zur Behandlung der rheumatoiden Arthritis, einzig Chloroquin steht zur Verfügung. Es wird ein Plan zum Ausschleichen der Steroide und initiieren der Chloroquin-Behandlung erstellt.

Bei einem Säugling mit Brechdurchfall gelingt es nicht, eine Infusion zu legen. Die perorale Substitution genügt nicht, das Kind verstirbt. Es zeigt sich, dass auch das Management und die Dokumentation mangelhaft waren.

Eine ganze Familie sitzt um das Bett einer Frau mit paretischen Beinen, sie ist 27 und hat 7 Kinder. Sie sind mausarm und haben weder Geld für Geflügel noch Fleisch noch regelmässig Gemüse; die Familie ernährt sich von Reis. Das Gesamtbild passt am besten zu einem nutritiven Vitamin-B-Mangel, beweisen können wir das jedoch nicht, da keine entsprechende Laboruntersuchung möglich ist. Die Familie kann - zumindest vorübergehend - dank Spenden in ein Ernährungsprogramm aufgenommen werden. Der Ehemann wird in einfachen physiotherapeutischen Massnahmen instruiert.

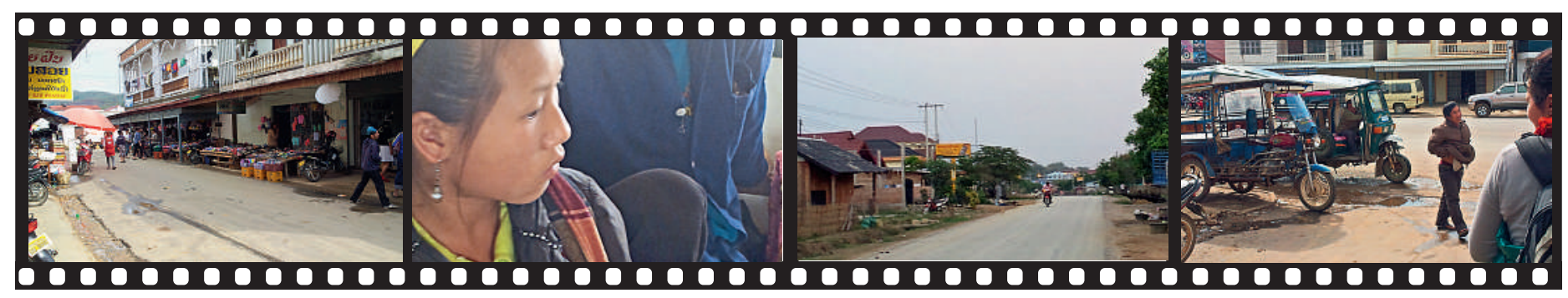




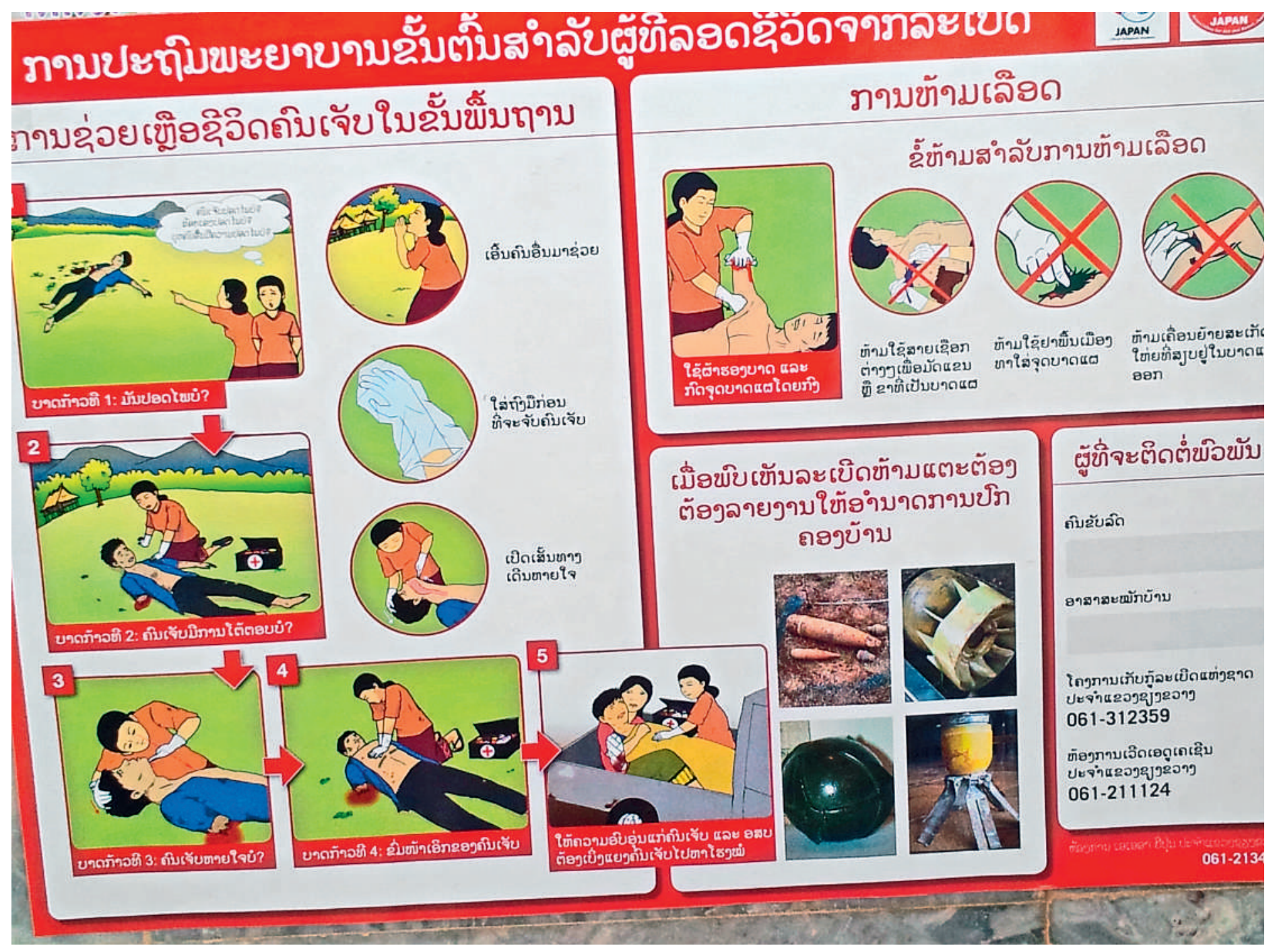

\section{Das Projekt und das Team}

Der Zürcher Gynäkologe Urs Lauper erhielt 2002 den Preis für humanitäre Projekte der SGGG für den Aufbau des Swiss Laos Hospital Project mit dem Partnerspital Mother and Child Hospital in Vientiane.

In der Rechtsform eines Vereins hat es sich in den letzten 10 Jahren weiterentwickelt und betreut in der Zwischenzeit neben gynäkologischen Projekten (Geburtensets für hygienisches Abnabeln, Ultraschallschulung usw.) auch zahlreiche Neonatologische Projekte in 10 Spitälern (Erneuerung der Neonatologien, Equipment, Schulung). Angeschlossen ist eine Spitalpartnerschaft zwischen dem Kantonsspital Winterthur und dem Provinzspital Phonsavanh.
2007 begründete der Hausarzt Beni Stutz zudem einen allgemeinärztlichen Track, in welchem die Möglichkeiten ausgelotet werden, einen Kleinspital in seiner Entwicklung zur Grundversorgung der lokalen Bevölkerung zu unterstützen.

Viermal pro Jahr verbringt mindestens eine Kollegln zwei bis drei Wochen im Projekt. Die Laborausstattung konnte so massiv verbessert, das Team permanent geschult werden. Die jungen AssistenzärztInnen sowie die «medical assistants» werden in der Sprechstunde und auf Stationsvisiten begleitet, Fälle diskutiert und nach Lösungen gesucht. Auf Basis dieser Fälle werden zwei bis drei Fortbildungen pro Woche abgehalten.

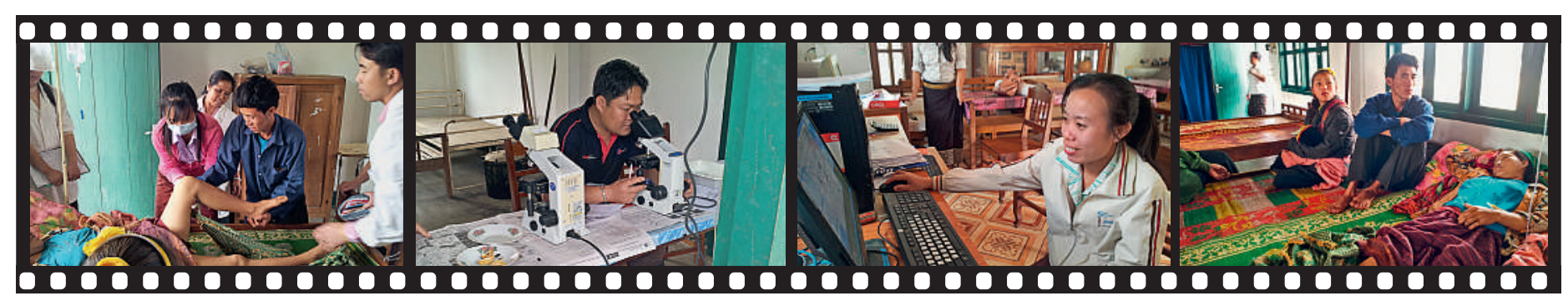


Ein Diabetes Care Team ist im Aufbau begriffen, bisher waren die Diabetiker nicht einmal im Spital registriert. Wir haben den Ultraschall eingeführt, den OP saniert, ein Projekt beschäftigt sich mit Hygiene und Berufskleidung, es wurden erste CPR-Kurse mit Schweizer Reanimations-Puppen durchgeführt.

Der Fächer von sinnvollen Aktivitäten ist riesig, wir haben eher das Problem, uns angemessen auf einige Schwerpunkte zu fokussieren. Seit kurzem gehören im Spital auch ein junger Gynäkologe und ein Anästhesist zum Team, das insgesamt rund 60 Personen umfasst.

\section{Die Einsätze}

Du fliegst von der Schweiz aus via Bangkok oder Hanoi in die laotische Hauptstadt Vientiane, von dort mit einem Inlandflug nach Phonsavanh, dann bringt Dich der Spitaltransporter nach Muang Kham. Seit 2012 gibt es dort ein Gästehaus des Projektes, in welchem Mitglieder von Hilfsequipen bei ihren Einsätzen angenehm wohnen können.

An einem Treffen mit dem Chefarzt Dr. Bounpheng werden die Schwerpunkte des aktuellen Einsatzes besprochen und Informationen über den Spital wie auch das Projekt ausgetauscht. Die Arbeitstage sind gut gefüllt mit Visiten, Schulungen, Bestandesaufnahmen in Labor, Apotheke usw. Gegessen wird im Gästehaus oder in einem der nahen Restaurants, eine Spitalküche gibt es nicht.

Am Abend bist Du müde; wenn nicht, kannst Du mit laotischen Teammitgliedern in den Ausgang, oder Du surfst im Internet oder bereitest eine Fortbildung vor etc. In der Nacht hast Du meistens Ruhe, es kann aber mal vorkommen, dass Du zu einem Patienten im septischen Schock gerufen wirst, falls die zuständigen Spezialisten nicht erreichbar sind. Hin und wieder steht ein Einsatz in einem Dorf an, wenn z.B. ein Spezialteam dort die Bevölkerung oder die Gesundheitsschwestern schult.

Wir tauchen somit tief in die buddhistische Kultur der Laoten, oft auch in die animistische Kultur anderer Volksgruppen ein. Die Einsätze sind extrem abwechslungsreich und spannend. Wir werden zur Beurteilung klinischer Situationen oft auf unser klinisches Grund- wissen und unsere Sinnesorgane zurückgeworfen, da wenig Zusatzuntersuchungen zur Verfügung stehen, ebenso ist die Verfügbarkeit therapeutischer Verfahren sehr beschränkt. Für die Einsätze steht ein Dolmetscher oder eine Dolmetscherin zur Verfügung.

Unser Kern-Team besteht aus zwei erfahrenen Hausärztinnen und drei ebensolchen Hausärzten. Gelegentlich werden wir begleitet von einer Fachfrau Gesundheit, einer Hebamme, einer Laborantin, einem Gynäkologen oder einem jungen hausärztlichen Kollegen. Die Einsätze sind ehrenamtlich, die Reisekosten werden jedoch übernommen.

Es ist oft sinnvoll, zu zweit dort zu sein. Arbeit gibt es genügend und der Austausch ist anregend und hilfreich. Wir freuen uns daher, wenn Dich das Projekt interessiert und Du gerne Näheres über einen Einsatz erfahren möchtest, insbesondere wenn Du Dir vorstellen kannst, diesen allenfalls in eine nachhaltige Projektbeteiligung zu überführen.

Weitere Informationen zum Gesamtprojekt findest Du auf www.swisslaos.ch, solche zum hausärztlichen Projekt kannst Du bei Interesse gerne bei einem von uns einholen:

Elisabeth Bandi-Ott: kbandi[at]bluewin.ch Kerstin Busch: buschkerstin [at] googlemail.com Andreas Graf: and.graf [at] bluewin.ch Beni Stutz: benjaminstutz [at] gmx.ch Marco Zoller: marco.zoller [at] hin.ch

Korrespondenz:

Dr. med. Marco Zoller

Facharzt Allgemeine Medizin FMH

Gemeinschaftspraxis am Meierhof

Limmattalstrasse 177

8049 Zürich

marco.zoller[at]hin.ch 